\title{
Selective Synthesis of Hexagonal Ag Nanoplates in a Solution-Phase Chemical Reduction Process
}

\author{
Mingzhu Liu ${ }^{1,2}$, Mei Leng ${ }^{1,2}$, Chao Yu ${ }^{1,2}$, Xin Wang ${ }^{3}$, and Cheng Wang ${ }^{1}(\bowtie)$ \\ ${ }^{1}$ State Key Laboratory of Rare Earth Resource Utilization, Changchun Institute of Applied Chemistry, Chinese Academy of Sciences, \\ Changchun, 130022, China \\ ${ }^{2}$ Graduate School of the Chinese Academy of Sciences, Beijing, 100049, China \\ ${ }^{3}$ Division of Chemical and Biomolecular Engineering, Nanyang Technological University, 637722, Singapore \\ Received: 8 August 2010 / Revised: 9 September 2010 / Accepted: 9 October 2010 \\ (C) The Author(s) 2010. This article is published with open access at Springerlink.com
}

\begin{abstract}
Two-dimensional (2-D) Ag nanoplates have surface plasmon resonances which can be tuned from the visible to the near-IR by varying the size and morphology of the nanoplates. Due to their anisotropic structures and different surface energy distributions, Ag nanoplates-especially triangular ones-are kinetically stable and can transform into other nanostructures. Taking advantage of the synergetic effects of $\mathrm{HNO}_{3}$ and $\mathrm{Cl}^{-}$in the reduction solution, uniform Ag hexagonal nanoplates (HNPs) have been captured during the transformation of $\mathrm{Ag}$ triangular nanoplates (TNPs). The dimensions of the Ag HNPs can be controlled by changing the concentrations of reagents in the reaction or/and reduction solutions. Resonance absorption spectra of the obtained Ag HNPs indicated that their in-plane resonance peaks could be tuned from the visible to the near-IR region, showing their potential applications in medical diagnosis.
\end{abstract}

\section{KEYWORDS}

Synthesis, Ag hexagonal nanoplates, etching effect, transformation

\section{Introduction}

Two-dimensional (2-D) Ag nanoparticles have attracted great research interest in the past decade due to their fascinating optical properties resulting from surface electrons interacting with incident light [1]. Among the multipolar resonant modes, the in-plane dipole resonance peak is found to be very intense and sensitive to the particle size and aspect ratio, as well as to the surrounding medium, which allows the extinction wavelength to be tuned from the visible to the near-IR region $[2,3]$. The sensitivity of the in-plane resonance peak to changes in the surrounding medium has been utilized in biological and chemical sensing of, e.g., proteins [4], polypeptides [5], anti-biotin [6] or carbohydrates [7]. The surface plasmons of an Ag nanoplate can also enhance the evanescent waves of a separated object. An Ag superlens can be fabricated when an Ag nanoplate of appropriate thickness is selected and the permittivity of the $\mathrm{Ag}$ and the adjacent object are equal and of opposite sign [8]. The resonant plasmons also result in a strong local surface-enhanced electromagnetic field [9], which can increase the scattering intensity of molecules

$\overline{\text { Address correspondence to cwang@ }}$ ciac.jl.cn 
adsorbed on Ag nanoplates and makes the Ag nanoplates good substrates for the detection of small amounts of material, or even single molecules, by surface enhanced Raman spectroscopy (SERS) [10, 11]. In addition, $\mathrm{Ag}$ nanoplates in solution can be also used as optical limiters, in which the nonlinear scattering of the Ag nanoplates has been proposed to play an important role in the optical limiting effects [12].

Generally there are three kinds of methods to synthesize Ag nanoplates [1] such as triangular (nanoprism), hexagonal and round (nanodisk) nanostructures. Nanosphere lithography (NSL) [13, 14], a physical technique, gives good control over shape, size and interparticle spacing but requires sophisticated instruments and is not amenable to scale up. Photoinduced growth [15-17], first reported by Jin et al. [15], has proved successful in the synthesis of triangular Ag nanoplates (TNPs) and has been extended to the preparation of hexagonal Ag nanoplates (HNPs) [18] or round Ag nanoplates [19] by transformation of Ag TNPs. To surmount the low yields and slow reaction rates of the photoinduced process, chemical solution reduction methods have been developed. In these methods, various reducing agents and organic additives have been investigated. The reducing reagents employed include hydrogen peroxide [20], sodium borohydride [20-22], ascorbic acid [23], hydrazine [24], poly(vinylpyrrolidone) (PVP) [25], and $N, N$-dimethylformamide (DMF) [26]. PVP [20, 26], sodium citrate [21,27], cetyltrimethyl ammonium bromide (CTAB) [22], and poly(sodium styrenesulfonate) (BSPP) [21] are among the organic additives which have been utilized in the reactions to protect the products from agglomeration and dictate their anisotropic growth. The products prepared by these solution-phase chemical reduction processes are usually Ag TNPs, which are always snipped at the corners, together with a small quantity of other 2-D as well as spherical Ag nanoparticles. The morphologies of the Ag nanoparticles are mainly determined by the initial geometry of nuclei formed at the beginning of the reaction, which is dependent on the intrinsic crystalline structure and the kinetic conditions employed [28]. Additionally, Ag nanoplates are thermodynamically unstable due to their high surface energies resulting from their large surface area [25].
Therefore the presence of structure defects and kinetic control of growth processes are prerequisites for the preparation of Ag nanoplates [23, 29-31]. Due to the high surface energies at the corners of $\mathrm{Ag}$ TNPs [32], Ag atoms at the corners easily dissolve and may re-deposit on the edges or flat faces of the nanoplates. Therefore Ag TNPs are usually snipped at the corners and can be easily transformed into hexagonal or/and round 2-D nanostructures. These transformations often occur simultaneously and products from different transformation stages always coexist in the reaction solution, which leads to a variety of shapes of $\mathrm{Ag}$ nanoparticles in the final products. Here we report a solution-phase chemical reduction method for the preparation of uniform Ag HNPs on a large scale at low temperature $\left(4-10^{\circ} \mathrm{C}\right)$ within $1-3 \mathrm{~h}$. When nitric acid $\left(\mathrm{HNO}_{3}\right)$ and chloride anions $\left(\mathrm{Cl}^{-}\right)$ are present in the reduction solution, the corner etching of Ag TNPs is greatly accelerated and occurs in a synchronous manner and uniform Ag HNPs can be captured as intermediates in the transformation process. Compared with previous reports of the preparation of similar Ag HNPs, in which a long photoinduced transformation time and/or very dilute precursors (in both photoinduced [18] and chemical reduction [33] processes) are required, our method provides a better control over the shape uniformity and tuning of particle size (from $40 \mathrm{~nm}$ to $350 \mathrm{~nm}$ in edge length) of Ag HNPs.

\section{Experimental}

\subsection{Synthesis of Ag HNPs}

Silver nitrate $\left(\mathrm{AgNO}_{3}\right)$, poly(vinylpyrrolidone) (PVP$\mathrm{K} 30,2.0 \mathrm{~mol} / \mathrm{L}$ in terms of the repeat unit, $M_{\mathrm{w}} \approx 50000$ ) and ammonium chloride $\left(\mathrm{NH}_{4} \mathrm{Cl}\right)$ were provided by Sinopharm Chemical Reagent Co., Ltd. Hydrazine hydrate $\left(\mathrm{N}_{2} \mathrm{H}_{4} \cdot \mathrm{H}_{2} \mathrm{O}\right)$ and $\mathrm{HNO}_{3}$, were purchased from Beijing Chemical Works. All reagents were used as received. Aqueous solutions of these reagents were prepared with ultrapure water, which was produced using an Ultrapure Water System (Purifier) and had resistivity larger than $18.0 \mathrm{M} \Omega$. In a typical process, $5.0 \mathrm{~mL}$ of a precursor solution containing $0.01 \mathrm{~mol} / \mathrm{L}$ $\mathrm{AgNO}_{3}, 5 \times 10^{-6} \mathrm{~mol} / \mathrm{L} \mathrm{NH}_{4} \mathrm{Cl}$ and $0.01 \mathrm{~mol} / \mathrm{L} \mathrm{PVP}$ was 
prepared and added into a $25-\mathrm{mL}$ flask. The flask was kept in an ice-water bath to maintain a low temperature between 4 and $10{ }^{\circ} \mathrm{C}$. To this flask was added $2.0 \mathrm{~mL}$ of a freshly prepared mixture of $1.4 \mathrm{~mL}$ water, $0.2 \mathrm{~mL}$ $\mathrm{N}_{2} \mathrm{H}_{4} \cdot \mathrm{H}_{2} \mathrm{O}$ aqueous solution $(0.2 \mathrm{~mol} / \mathrm{L}), 0.2 \mathrm{~mL}$ $\mathrm{NH}_{4} \mathrm{Cl}$ aqueous solution $(0.5 \mathrm{mmol} / \mathrm{L})$ and $0.2 \mathrm{~mL}$ $\mathrm{HNO}_{3}$ aqueous solution $(0.1 \mathrm{~mol} / \mathrm{L})$. This mixture is known as the reduction solution. The reduction solution was added by means of a peristaltic pump at a speed of $0.1 \mathrm{~mL} / \mathrm{min}$. The aqueous solution in the flask was allowed to react for $1 \mathrm{~h}$ after the introduction of the reduction solution. The resulting mixture was transferred to a centrifugation tube and centrifuged at $4000 \mathrm{r} / \mathrm{min}$ for $20 \mathrm{~min}$. The product was washed three times by ultrapure water followed by centrifugation. For UV-vis characterization, the product was redispersed in water.

\subsection{Characterization}

X-ray diffraction (XRD) pattern was recorded with a Bruker D8 Focus diffractometer using $\mathrm{Cu} \mathrm{K} \alpha$ radiation $(40 \mathrm{kV}, 40 \mathrm{~mA})$ with a scanning speed of $10^{\circ} / \mathrm{min}$. Transmission electron microscopy (TEM) images and ED patterns were obtained using a FEI Tecnai F20 instrument operated at $200 \mathrm{kV}$. Field emission scanning electron microscopy (FESEM) images were taken using a Hitachi S-4800 scanning electron microscope operated at an acceleration voltage of $10 \mathrm{kV}$. UV-vis spectra were recorded on a HP UV-91 spectrophotometer.

\section{Results and discussion}

As shown in Figs. 1(a) and 1(b), the Ag HNPs obtained from the typical synthetic process using the above parameters have an average edge length and thickness of $60 \mathrm{~nm}$ and $20 \mathrm{~nm}$ respectively. A panorama view of the products under lower magnification is provided in Fig. S-1 in the Electronic Supplementary Material (ESM). The XRD pattern (Fig. 1(c)) is dominated by the $<111>$ peak, which indicates that the HNPs are mainly bound by the (111) planes. Two additional peaks adjacent to the $<111>$ peak can be also observed in the magnified pattern (inset in Fig. 1(c)). These two peaks have been ascribed to hexagonal structural defects in Ag nanoplates [34]. TEM (Fig. 1(d)) shows that a single Ag HNP is slightly truncated at its six corners. The hexagonal symmetry of the scattered spots in the ED pattern (Fig. 1(e)) also suggests that the surfaces of HNPs are bounded by (111) planes. The presence of formally forbidden (1/3) 4422$\}$ spots in the ED and associated lattice fringes with a spacing of $2.5 \AA$ in the high-resolution TEM (HRTEM) image (Fig. 1(f)) are consistent with the presence of structural defects parallel to the flat (111) surface [34].

The size of the Ag HNPs can be tuned by simply changing the concentrations of reagents in the precursor or/and reduction solution. Ag HNPs with an average edge length of around $40 \mathrm{~nm}$ were obtained when the concentrations of $\mathrm{AgNO}_{3}$ and PVP were reduced to half of the values used in the typical process (Fig. 2(a)). Larger nanoplates $(60-350 \mathrm{~nm}$ in edge length) with a broad length distribution were
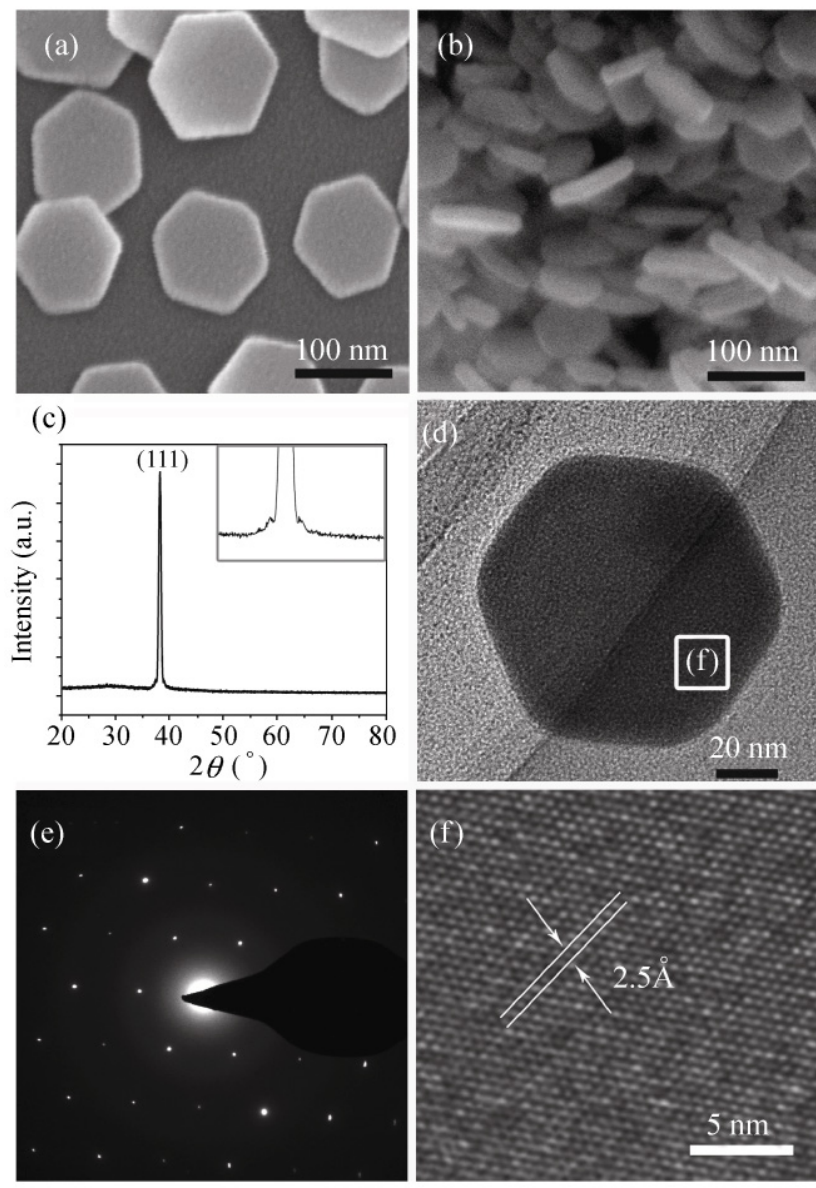

Figure 1 Structural characterization of Ag HNPs obtained from the typical process: $(a, b)$ FESEM images; (c) XRD pattern with the inset showing two additional peaks adjacent to the (111) diffraction peak; (d) TEM image; (e) ED pattern and (f) HRTEM image taken from the marked area in (d) 

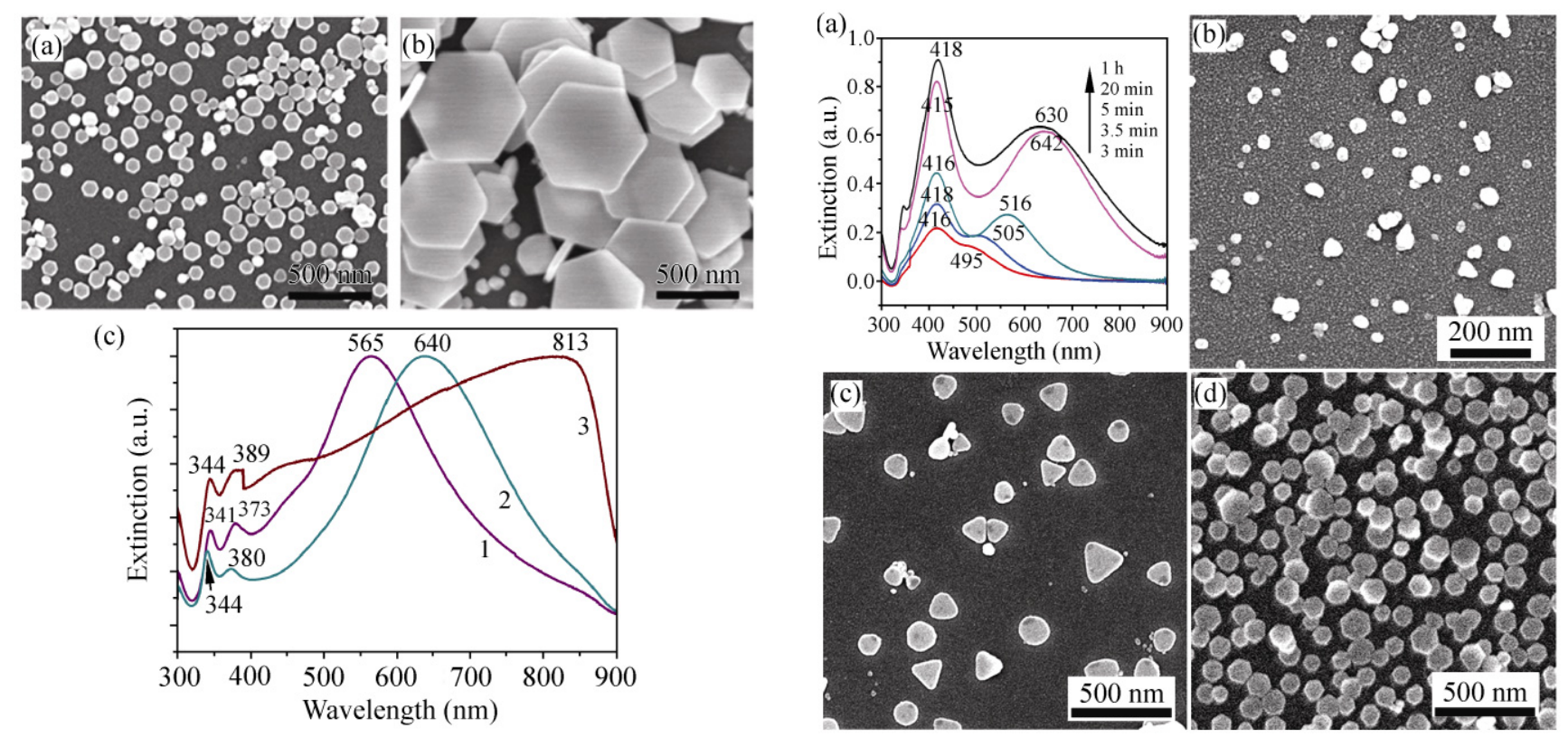

Figure 2 FESEM images of Ag HNPs with different edge lengths: (a) $40 \mathrm{~nm}$; (b) 60-350 nm; (c) UV-vis spectra of Ag HNPs where curves 1,2 , and 3 correspond to nanoplates with edge lengths of 40,60 and $60-350 \mathrm{~nm}$

produced with higher concentrations of reagents in both the precursor and reduction solutions (Fig. 2(b)). Details of the experimental conditions corresponding to the samples displayed in Figs. 1(a), 2(a) and 2(b) are listed in Table S-1 in the ESM. Figure 2(c) shows the UV-vis spectra of the three samples with different edge lengths. The in-plane dipole plasmon resonance peaks show a marked red-shift from $565 \mathrm{~nm}$ to $813 \mathrm{~nm}$ with increasing edge length. The slight red-shifts of the out-plane quadrupole and dipole plasmon resonance modes [15], from $341 \mathrm{~nm}$ to $344 \mathrm{~nm}$ and $373 \mathrm{~nm}$ to $389 \mathrm{~nm}$ respectively, could be indicative of a slight increase in the thickness of the nanoplates [3].

To study the morphological evolution, $0.5 \mathrm{~mL}$ aliquots of the reaction solution were removed from the synthesis mixture after different reaction times. These solutions were analyzed by UV-vis absorption spectroscopy (Fig. 3(a)) and the morphologies were studied by SEM (Figs. 3(b)-3(d)). Three minutes after commencing addition of the reduction solution, the mixture turned yellow and then rapidly became red. This solution gave a single broad plasmon peak centered about $416 \mathrm{~nm}$ with a shoulder peak at $495 \mathrm{~nm}$ and contained small $\mathrm{Ag}$ nanoparticles as well as a minor amount of triangular plates (Fig. 3(b)). On

Figure 3 (a) Time-dependent UV-vis spectra of the reaction solution and FESEM images of samples formed after different reaction times: (b) $5 \mathrm{~min}$; (c) $20 \mathrm{~min}$; (d) $1 \mathrm{~h}$

further reaction, the red solution turned green and remained this color until addition of the reduction solution was complete. The green solution showed a peak due to the out-plane quadrupole plasmon resonance. During the course of the reaction, the peak position of the in-plane dipole resonance shifted from 495 to $642 \mathrm{~nm}$ accompanied by increases in the intensities of both dipole resonances. FESEM images showed that the green solution contains predominantly slightly corner-truncated Ag TNPs (Fig. 3(c)). On further incubating the reaction solution, a brown color appeared about $10 \mathrm{~min}$ after the completion of the addition of the reduction solution. The green-brown solution subsequently became blue-brown within $30 \mathrm{~min}$ and this color remained unchanged over the following $2 \mathrm{~h}$. The bicolor appearance can be ascribed to the strong scattering of larger Ag nanoparticles, which is believed to lead to a significant difference in reflection and transmission [35]. FESEM images indicated that hexagonal silver nanoplates made up $>80 \%$ of the product (Fig. $3(\mathrm{~d}$ )). Along with the morphological transformation from triangular to hexagonal nanoplates, the in-plane dipole plasmon peak showed a slight blue-shift (Fig. 3(a)). The corner truncation of TNPs and particle size increase of HNPs might be responsible for this 
slight shift in plasmon resonance. It should be noted that the observed in-plane dipole plasmon resonance for the Ag HNPs differs from that of the typical synthetic batch (Fig. 2(c)) as they were obtained in different batches. Further incubation of the Ag HNPs should be avoided because it causes further truncations at their corners and leads to the formation of some round disks as shown in Figs. 4(a) and 4(b). However, the Ag HNPs are fairly stable (they remain unchanged for at least one month) when separated from the mother liquor.

The time-dependent morphological evolution of silver nanostructures (Fig. 3) clearly shows that Ag HNPs are obtained by transformation of Ag TNPs. The transformation from Ag TNPs to HNPs may involve two processes, similar to those observed in the photoconversion of TNPs [18]. One is the oxidation of $\mathrm{Ag}$ atoms at the corner of the TNPs followed by their dissolution into the reaction solution due to their higher surface energy. The other is the reduction of $\mathrm{Ag}^{+}$in the reaction solution by excess $\mathrm{N}_{2} \mathrm{H}_{4}$. The newly formed $\mathrm{Ag}$ atoms tend to be adsorbed by sites with high surface energy, which, in this case, are likely to be the side facets of $\mathrm{Ag}$ nanoplates having structural defects.

The formation and subsequent transformation of Ag TNPs were found to be very sensitive to the experimental conditions. Deviation from the optimized experimental conditions resulted in dramatic morphological variations; typically the yield of HNPs was low, as TNPs and spherical nanoparticles were also formed. Among the parameters investigated, the concentration of $\mathrm{Cl}^{-}$seems to play a pivotal role in the formation of Ag HNPs. The effect on the synthesis

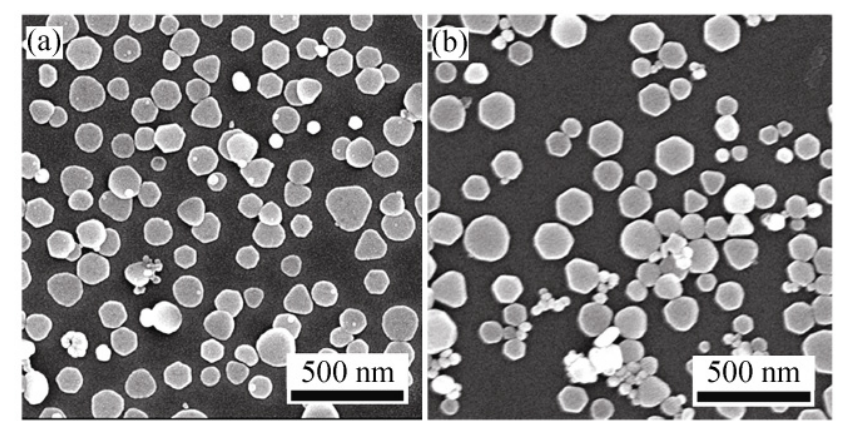

Figure 4 FESEM images of products obtained after being incubated in the mother liquor for (a) $3 \mathrm{~h}$ and (b) $24 \mathrm{~h}$ of $\mathrm{Ag}$ nanoparticles of varying the $\mathrm{Cl}^{-}$concentration was investigated, with all other parameters the same as those in the typical process. If there was no $\mathrm{Cl}^{-}$in the reaction system (in neither the precursor nor reduction solutions), FESEM (Fig. 5(a)) showed that very few Ag TNPs or HNPs were formed after complete addition of the reduction solution and aging the mixture for $40 \mathrm{~min}$. When $\mathrm{Cl}^{-}$was present in the precursor solution only (with a concentration of $\left.5.0 \times 10^{-6} \mathrm{~mol} / \mathrm{L}\right), \mathrm{Ag}$ TNPs were the main product (Fig. 5(b)). A further increase in the concentration of $\mathrm{Cl}^{-}$in the precursor solution should be avoided because more spherical Ag nanoparticles were produced (Fig. S-2 in the ESM). However, higher quantities of $\mathrm{Cl}^{-}$in the reaction system can be tolerated by introducing additional $\mathrm{Cl}^{-}$in the reduction solution. When the concentration of additional $\mathrm{Cl}^{-}$in the reduction solution was varied from 0.02 to $0.08 \mathrm{mmol} / \mathrm{L}$ (Fig. 1(a) and Figs. 5(c)-5(e)), the products were predominantly Ag HNPs. Further increasing the concentration of additional $\mathrm{Cl}^{-}$in the reduction solution to $1.0 \mathrm{mmol} / \mathrm{L}$ slowed down the reduction of $\mathrm{Ag}^{+}$, and $\mathrm{Ag}$ nanoparticles with various morphologies were obtained as shown in Fig. 5(f). The effect of adding $\mathrm{Cl}^{-}$to the reduction solution when there was no $\mathrm{Cl}^{-}$in the precursor solution was also investigated. If the amounts of $\mathrm{Cl}^{-}$in the reduction solution were comparable to the combined amounts of $\mathrm{Cl}^{-}$in the reaction and reduction solutions which afforded $\mathrm{Ag}$ TNPs and HNPs in the aforementioned processes, particles with similar morphologies were obtained but the size distributions tended to be broader (results not shown). The UV-vis spectra of Ag HNPs obtained with different amount of $\mathrm{Cl}^{-}$are shown in Fig. 6 . With increasing concentration of $\mathrm{Cl}^{-}$, the in-plane dipole resonance showed a blue-shift, which implies a decrease in particle size. Meanwhile, the two outplane plasmon resonance peaks showed a gradual red-shift with increasing $\mathrm{Cl}^{-}$concentration, suggesting that the thickness of the nanoplates increases [3]. This provides another mean of tuning the particle size, but the effects are much less dramatic than those obtained by varying the concentrations of $\mathrm{AgNO}_{3}$ and PVP (shown in Fig. 2). Any influence of the cations in the chloride salts can be excluded since replacing $\mathrm{NH}_{4} \mathrm{Cl}$ 


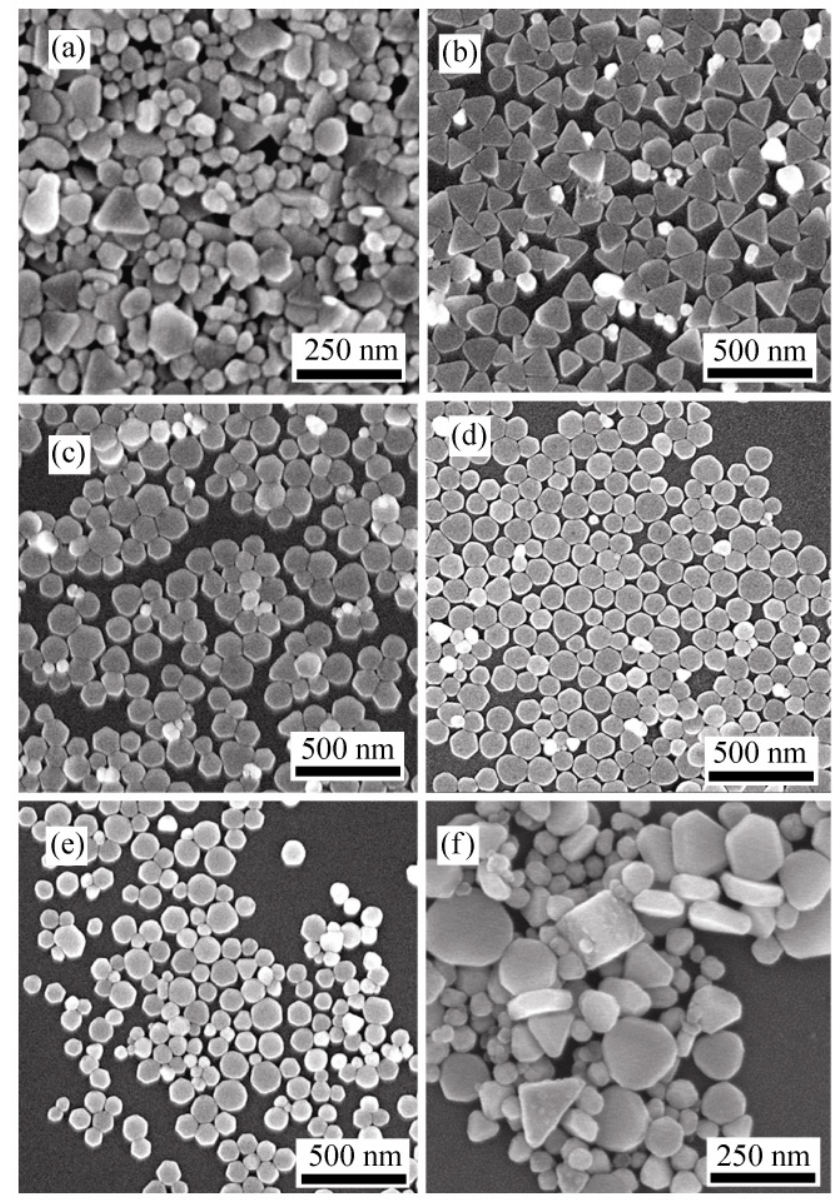

Figure 5 FESEM images of the products obtained (a) without $\mathrm{Cl}^{-}$in the reaction system, and with $5.0 \times 10^{-6} \mathrm{~mol} / \mathrm{L} \mathrm{Cl}^{-}$in the precursor solution and (b) $0 \mathrm{~mol} / \mathrm{L}$, (c) $2.0 \times 10^{-5} \mathrm{~mol} / \mathrm{L}$, (d) $3.0 \times$ $10^{-5} \mathrm{~mol} / \mathrm{L}$, (e) $8.0 \times 10^{-5} \mathrm{~mol} / \mathrm{L}$, and (f) $1.0 \times 10^{-3} \mathrm{~mol} / \mathrm{L}$ in the reduction solution

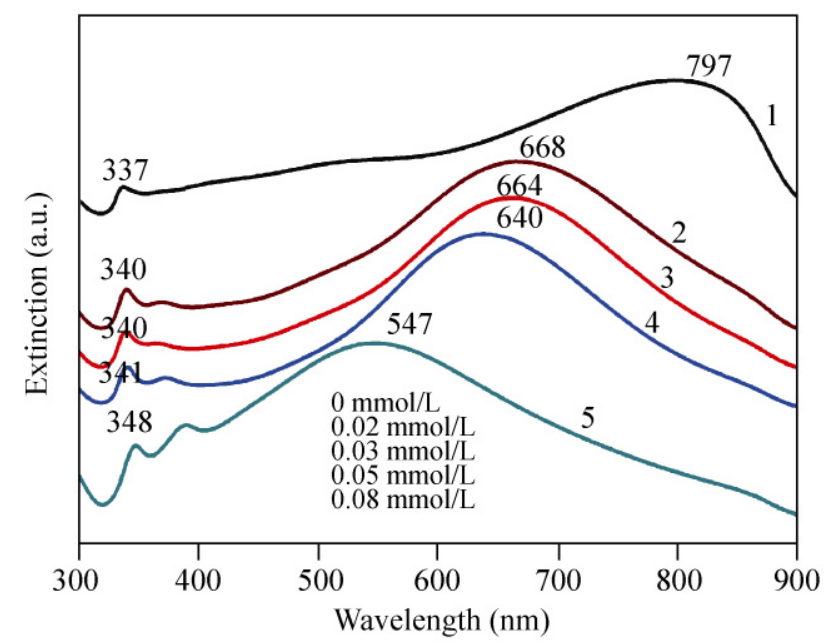

Figure 6 UV-vis spectra of the Ag HNPs prepared with different concentration of $\mathrm{Cl}^{-}$in the reduction solution by $\mathrm{NaCl}$ in the reduction solution while keeping the other reaction parameters fixed afforded Ag HNPs with similar dimensions and surface plasmon resonances. This confirms that $\mathrm{Cl}^{-}$influences the morphology of the 2-D Ag nanoplates. On the basis of the above experimental results, three conclusions can be drawn: (1) $\mathrm{Cl}^{-}$favors the formation of 2-D Ag nanostructures; (2) Ag TNPs can be formed when relatively low amounts of $\mathrm{Cl}^{-}$are present in either the precursor or the reduction solution; (3) Higher concentrations of $\mathrm{Cl}^{-}$are required to transform $\mathrm{Ag}$ TNPs into HNPs and continuous addition of $\mathrm{Cl}^{-}$favors the transformation.

Akin to citrate [36], hydrazine has a higher reduction activity at high $\mathrm{pH}$ values. Directly adding hydrazine solution to the $\mathrm{AgNO}_{3}$ solution containing PVP (the original $\mathrm{pH}$ value was about 4.7 ) led to an increase in the $\mathrm{pH}$ of the reaction system, due to the alkaline nature of hydrazine, itself and afforded spherical Ag nanoparticles. This fast reaction can be suppressed by introducing an acid into the reduction solution, and $\mathrm{HNO}_{3}$ was found to be a good choice. The optimum $\mathrm{pH}$ of the reduction solution was found to be in the range 7.8-8.2. It should be pointed out that changing the amount of $\mathrm{HNO}_{3}$ is not advisable as the $\mathrm{pH}$ value will vary accordingly. Therefore care should be taken in preparing the reduction solution. On continuous introduction of the reduction solution by a peristaltic pump, the $\mathrm{pH}$ value in the reaction solution decreased and eventually reached around 3.5. The decreased $\mathrm{pH}$ value implies an increase of $\mathrm{HNO}_{3}$ generated in the reaction solution from $\mathrm{AgNO}_{3}$, which might dissolve the silver nanoparticles that were initially formed. A similar phenomenon has been previously observed in the preparation of $\mathrm{Ag}$ nanocubes in a polyol synthesis and the underlying mechanism was rationalized [37]. We suggest that the reduced $\mathrm{pH}$ value and in situ generation of $\mathrm{HNO}_{3}$, together with the presence of appropriate amounts of $\mathrm{Cl}^{-}$, might facilitate the selective etching of Ag TNPs and their subsequent transformation to Ag HNPs.

The addition of an appropriate amount of PVP is indispensable to prevent the aggregation of the nuclei in the early reaction stages as demonstrated by Xia et al. [38]. However, varying the molar ratio of PVP to $\mathrm{AgNO}_{3}$ within the range 0.5-2.0 did not significantly 
alter the morphology of the product. In addition, the reaction temperature should be controlled between 4 and $10{ }^{\circ} \mathrm{C}$ in order to achieve a favorable reaction rate and produce uniform Ag HNPs. Using a peristaltic pump to add the reduction solution to the reaction solution allows the separation of nucleation and subsequent particle growth processes, as shown by the FESEM images in Fig. 3, and offers a better control over morphology and particle size distribution.

Despite being enclosed by low-index facets, Ag TNPs and HNPs have high surface energies due to their high surface area and are kinetically stable products. This has been verified by many studies of photoinduced and solution reduction processes. The arrest of nearly pure Ag TNPs has been demonstrated in many previous reports, but this is not the case for $\mathrm{Ag}$ HNPs. The slow rate of transformation of $\mathrm{Ag}$ TNPs to HNPs is likely to be responsible for the failure to arrest Ag HNPs, since the transformation of $\mathrm{Ag}$ HNPs to round shapes occurs before the completion of the transformation from TNPs. The mechanism of formation of Ag TNPs still remains an open controversial issue. The adsorption of organic additives on specific crystalline facets has been invoked to interpret the morphological evolution of 2-D Ag nanostructures in both photoinduced and solution reduction processes $[23,38,39]$. However, the negligible effect on the 2-D morphologies of varying the molar ratio of $\mathrm{PVP}$ to $\mathrm{AgNO}_{3}$ suggests that this mechanism is not applicable in our case. Recently, it has become fairly clear that considerable twinning or stacking faults are present in 2-D Ag nanoplates. These faults tend to occur at the thinner sides of the nanocrystals and serve as the preferred growth sites. As a result, 2-D nanostructures are formed, and growth along the $<111>$ direction is hindered. This is supported by our indirect observations using XRD, TEM, and ED. Therefore it is likely that the fault-induced mechanism dictates the formation of Ag TNPs in our synthesis. In addition, the transformation of Ag TNPs to HNPs is believed to be accelerated by $\mathrm{Cl}^{-}$. The etching effect of halide anions on nanostructures of noble metals has been well documented in the literature [40,41]. Such accelerated etching of Ag TNPs allows the capture of Ag HNPs during the transformation of 2-D Ag nanoplates.

\section{Conclusions}

Uniform Ag HNPs can be obtained via a solutionphase chemical reduction process. Experimental results revealed that appropriate nucleation and growth rates favoring the formation of $\mathrm{Ag}$ nanoplates could be achieved by finely tuning the experimental conditions. Among these parameters, the concentrations of $\mathrm{HNO}_{3}$ and $\mathrm{Cl}^{-}$in the reduction solution as well as the mode of addition of $\mathrm{Cl}^{-}$were found to play critical roles in the etching process of Ag TNPs. By tuning their dimensions, Ag HNPs exhibiting resonance absorption spectra from the visible to the near-IR range can be obtained. XRD, ED and HRTEM data suggest that the fault-induced growth mechanism may dictate the growth of 2-D nanostructures in our process.

\section{Acknowledgements}

This work is supported by the National Basic Research Program of China (No. 2007CB925101) and the National Natural Science Foundation of China (NSFC) Fund for Creative Research Groups (No. 20921002).

Electronic Supplementary Material: A panorama of Ag HNPs prepared from a typical synthesis, details of the experimental conditions corresponding to the samples displayed in Fig. 1(a), Fig. 2(a), and Fig. 2(b) and a FESEM image of samples prepared with a higher $\mathrm{Cl}^{-}$concentration in the precursor solution are available in the online version of this article at http: //dx.doi.org/10.1007/s12274-010-0055-z and accessible free of charge.

Open Access: This article is distributed under the terms of the Creative Commons Attribution Noncommercial License which permits any noncommercial use, distribution, and reproduction in any medium, provided the original author(s) and source are credited.

\section{References}

[1] Pastoriza-Santos, I.; Liz-Marzan, L. M. Colloidal silver nanoplates. State of the art and future challenges. J. Mater. Chem. 2008, 18, 1724-1737.

[2] Charles, D. E.; Aherne, D.; Gara, M.; Ledwith, D. M.; Gun'ko, Y. K.; Kelly, J. M.; Blau, W. J.; Brennan-Fournet,

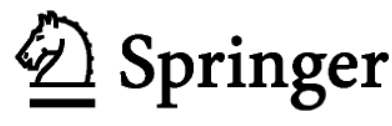


M. E. Versatile solution phase triangular silver nanoplates for highly sensitive plasmon resonance sensing. ACS Nano 2009, 4, 55-64.

[3] Brioude, A.; Pileni, M. P. Silver nanodisks: Optical properties study using the discrete dipole approximation method. $J$. Phys. Chem. B 2005, 109, 23371-23377.

[4] Haes, A. J.; Van Duyne, R. P. A nanoscale optical biosensor: Sensitivity and selectivity of an approach based on the localized surface plasmon resonance spectroscopy of triangular silver nanoparticles. J. Am. Chem. Soc. 2002, 124, 1059610604.

[5] Malinsky, M. D.; Kelly, K. L.; Schatz, G. C.; Van Duyne, R $P$. Chain length dependence and sensing capabilities of the localized surface plasmon resonance of silver nanoparticles chemically modified with alkanethiol self-assembled monolayers. J. Am. Chem. Soc. 2001, 123, 1471-1482.

[6] Riboh, J. C.; Haes, A. J.; McFarland, A. D.; Ranjit Yonzon, C.; Van Duyne, R. P. A nanoscale optical biosensor: Realtime immunoassay in physiological buffer enabled by improved nanoparticle adhesion. J. Phys. Chem. B 2003, 107, 1772-1780.

[7] Yonzon, C. R.; Jeoung, E.; Zou, S.; Schatz, G. C.; Mrksich, M.; Van Duyne, R. P. A Comparative analysis of localized and propagating surface plasmon resonance sensors: The binding of concanavalin A to a monosaccharide functionalized self-assembled monolayer. J. Am. Chem. Soc. 2004, 126, 12669-12676.

[8] Fang, N.; Lee, H.; Sun, C.; Zhang, X. Sub-diffraction-limited optical imaging with a silver superlens. Science 2005, 308, 534-537.

[9] Kelly, K. L.; Coronado, E.; Zhao, L. L.; Schatz, G. C. The optical properties of metal nanoparticles: The influence of size, shape, and dielectric environment. J. Phys. Chem. B 2003, 107, 668-677.

[10] Cui, B.; Clime, L.; Li, K.; Veres, T. Fabrication of large area nanoprism arrays and their application for surface enhanced Raman spectroscopy. Nanotechnology 2008, 19, 145302.

[11] Wang, Y.; Zou, X.; Ren, W.; Wang, W.; Wang, E. Effect of silver nanoplates on Raman spectra of $p$-aminothiophenol assembled on smooth macroscopic gold and silver surface. J. Phys. Chem. C 2007, 111, 3259-3265.

[12] Lakshminarayana, P.; Qing-Hua, X.; Mohan, S. D.; Wei, J. Optical limiting properties of silver nanoprisms. Appl. Phys. Lett. 2008, 92, 263110.

[13] Jensen, T. R.; Duval, M. L.; Kelly, K. L.; Lazarides, A. A.; Schatz, G. C.; Van Duyne, R. P. Nanosphere lithography: Effect of the external dielectric medium on the surface plasmon resonance spectrum of a periodic array of sliver nanoparticles. J. Phys. Chem. B 1999, 103, 9846-9853.
[14] Jensen, T. R.; Malinsky, M. D.; Haynes, C. L.; Van Duyne, R. P. Nanosphere lithography: Tunable localized surface plasmon resonance spectra of silver nanoparticles. J. Phys. Chem. B 2000, 104, 10549-10556.

[15] Jin, R. C.; Cao, Y. W.; Mirkin, C. A.; Kelly, K. L.; Schatz, G. C.; Zheng, J. G. Photoinduced conversion of silver nanospheres to nanoprisms. Science 2001, 294, 1901-1903.

[16] Callegari, A.; Tonti, D.; Chergui, M. Photochemically grown silver nanoparticles with wavelength-controlled size and shape. Nano Lett. 2003, 3, 1565-1568.

[17] Jin, R. C.; Cao, Y. C.; Hao, E. C.; Metraux, G. S.; Schatz, G. C.; Mirkin, C. A. Controlling anisotropic nanoparticle growth through plasmon excitation. Nature 2003, 425, 487-490.

[18] An, J.; Tang, B.; Ning, X.; Zhou, J.; Zhao, B.; Xu, W.; Corredor, C.; Lombardi, J. R. Photoinduced shape evolution: From triangular to hexagonal silver nanoplates. J. Phys. Chem. C 2007, 111, 18055-18059.

[19] Zhang, Q.; Ge, J. P.; Pham, T.; Goebl, J.; Hu, Y. X.; Lu, Z.; Yin, Y. D. Reconstruction of silver nanoplates by UV irradiation: Tailored optical properties and enhanced stability. Angew. Chem. Int. Ed. 2009, 48, 3516-3519.

[20] Metraux, G. S.; Mirkin, C. A. Rapid thermal synthesis of silver nanoprisms with chemically tailorable thickness. $A d v$. Mater. 2005, 17, 412-415.

[21] Xue, C.; Mirkin, C. A. pH-switchable silver nanoprism growth pathways. Angew. Chem. Int. Ed. 2007, 46, 2036-2038.

[22] Chen, S.; Fan, Z.; Carroll, D. L. Silver nanodisks: Synthesis, characterization, and self-assembly. J. Phys. Chem. B 2002, 106, 10777-10781.

[23] Chen, S. H.; Carroll, D. L. Synthesis and characterization of truncated triangular silver nanoplates. Nano Lett. 2002, 2, 1003-1007.

[24] Maillard, M.; Giorgio, S.; Pileni, M. P. Silver nanodisks. $A d v$. Mater. 2002, 14, 1084-1086.

[25] Washio, I.; Xiong, Y. J.; Yin, Y. D.; Xia, Y. N. Reduction by the end groups of poly(vinyl pyrrolidone): A new and versatile route to the kinetically controlled synthesis of $\mathrm{Ag}$ triangular nanoplates. Adv. Mater. 2006, 18, 1745-1749.

[26] Pastoriza-Santos, I.; Liz-Marzan, L. M. Synthesis of silver nanoprisms in DMF. Nano Lett. 2002, 2, 903-905.

[27] Jia, H. Y.; Zeng, J. B.; An, J.; Song, W.; Xu, W. Q.; Zhao, B. Preparation of triangular and hexagonal silver nanoplates on the surface of quartz substrate. Thin Solid Films 2008, 516, 5004-5009.

[28] Wiley, B.; Sun, Y.; Xia, Y. Synthesis of silver nanostructures with controlled shapes and properties. Acc. Chem. Res. 2007, 40, 1067-1076.

[29] C. Lofton, W. S. Mechanisms controlling crystal habits of gold and silver colloids. Adv. Funct. Mater. 2005, 15, 1197- 1208. 
[30] Aherne, D.; Ledwith, D. M.; Gara. M.; Kelly, J. M. Optical properties and growth aspects of silver nanoprisms produced by a highly reproducible and rapid synthesis at room temperature. Adv. Funct. Mater. 2008, 18, 2005-2016.

[31] Germain, V.; Li, J.; Ingert, D.; Wang, Z. L.; Pileni, M. P. Stacking faults in formation of silver nanodisks. J. Phys. Chem. B 2003, 107, 8717-8720.

[32] Wang, Z. L. Transmission electron microscopy of shapecontrolled nanocrystals and their assemblies. J. Phys. Chem. B 2000, 104, 1153-1175.

[33] Cao, Z. W.; Fu, H. B.; Kang, L. T.; Huang, L. W.; Zhai, T. Y.; Ma, Y.; Yao, J. N. Rapid room-temperature synthesis of silver nanoplates with tunable in-plane surface plasmon resonance from visible to near-IR. J. Mater. Chem. 2008, 18, 2673-2678.

[34] Rocha, T. C. R.; Zanchet, D. Structural defects and their role in the growth of Ag triangular nanoplates. J. Phys. Chem. C 2007, 111, 6989-6993.

[35] Pietrobon, B.; Kitaev, V. Photochemical synthesis of monodisperse size-controlled silver decahedral nanoparticles and their remarkable optical properties. Chem. Mater. 2008, 20, 5186-5190.
[36] Dong, X.; Ji, X.; Wu, H.; Zhao, L.; Li, J.; Yang, W. Shape control of silver nanoparticles by stepwise citrate reduction. J. Phys. Chem. C 2009, 113, 6573-6576.

[37] Im, S. H.; Lee, Y. T.; Wiley, B.; Xia, Y. N. Large-scale synthesis of silver nanocubes: The role of $\mathrm{HCl}$ in promoting cube perfection and monodispersity. Angew. Chem. Int. Ed. 2005, 44, 2154-2157.

[38] Sun, Y. A.; Xia, Y. N. Triangular nanoplates of silver: Synthesis, characterization, and use as sacrificial templates for generating triangular nanorings of gold. Adv. Mater. 2003, 15, 695-699.

[39] Tang, B.; Xu, S. P.; An, J.; Zhao, B.; Xu, W. Q. Photoinduced shape conversion and reconstruction of silver nanoprisms. $J$. Phys. Chem. C 2009, 113, 7025-7030.

[40] An, J.; Tang, B.; Zheng, X. L.; Zhou, J.; Dong, F. X.; Xu, S. P.; Wang, Y.; Zhao, B.; Xu, W. Q. Sculpturing effect of chloride ions in shape transformation from triangular to discal silver nanoplates. J. Phys. Chem. C 2008, 112, 15176-15182.

[41] Cathcart, N.; Frank, A. J.; Kitaev, V. Silver nanoparticles with planar twinned defects: Effect of halides for precise tuning of plasmon resonance maxima from 400 to $>900 \mathrm{~nm}$. Chem. Commun. 2009, 7170-7172. 